\title{
Free in-plane vibration of super-elliptical plates
}

\author{
Murat Altekin \\ Department of Civil Engineering, Yildiz Technical University, 34210 Esenler, Istanbul, Turkey \\ Tel.: +90 212 3835148; Fax: +90 212 3835133; E-mail: altekin@yildiz.edu.tr
}

Received 4 March 2008

Revised 17 April 2009

\begin{abstract}
Free in-plane vibration of super-elliptical plates of uniform thickness was investigated by the Ritz method. A large variety of plate shapes ranging from an ellipse to a rectangle were examined. Two cases were considered: (1) a completely free, and (2) a point-supported plate. The geometrical boundary conditions were satisfied by the Lagrange multipliers. The results were compared with those of rectangular plates. Basically good agreement was obtained. Matching results were reported, and the discrepancies were highlighted.
\end{abstract}

Keywords: Super-elliptical, plate, Ritz, in-plane, vibration

\section{Introduction}

In the vast literature devoted to the dynamic analysis of plates [1-38], transverse vibration has received significant attention and the solutions have been available for a wide range of shapes and boundary conditions [21]. This is probably partly because transverse motion is prone to excitation from external sources [16], and partly because the natural frequencies of plates in lateral vibration are generally much lower than those of plates in in-plane vibration [25]. However, in-plane modes can be practically important in aerospace structures [21], and in the design of ship hulls [24].

The studies on the in-plane vibration of plates have basically focused on isotropic rectangular plates solved numerically by various methods [1-8,21-25]. As far as the author knows the unique paper examining the inplane vibration of rectangular plates with intermediate point-supports was submitted by Kobayashi et al. [1]. They investigated the point-supported rectangular plates and presented the eigenvalues of a completely free plate as well. Bardell et al. [2] dealt with rectangular plates with either completely free or constrained edges. Higher modes of rectangular plates were presented by Hyde et al. [3]. An improved Fourier series method was utilized by Du et al. [4] to solve rectangular plates with elastically restrained edges. Gorman [5,6] employed the superposition method to analyze rectangular plates. Wang and Wereley [21] presented the solutions of a rectangular plate with constrained edges by the Kantorovich-Krylov method. Larsson [23] examined a free isotropic plate experimentally.

The present study was motivated by the scarcity of contributions on the in-plane vibrations of super-elliptical plates [30-38]. The fundamental (lowest) frequency was obtained and the optimum support location ${ }^{1}$ was searched. Two cases were handled: (1) a completely free plate, (2) a plate with symmetrically distributed four point-supports on the diagonals. The Ritz method - one of the most prevalent methods used in applied mechanics (e.g., Refs. [1-3]) was employed, and the geometrical boundary conditions were satisfied by the Lagrange multipliers. The results obtained in the present study were checked with those of rectangular plates. ${ }^{2}$ Almost identical values were found for the first case. Good agreement was obtained for SS modes for the second case. However, lower frequencies were reported for SA, and AA modes. The corresponding mode shapes were demonstrated.

\footnotetext{
${ }^{1}$ Optimum location of the support is defined by the support position which yields the highest fundamental frequency.

${ }^{2}$ The results obtained from Refs. [1-4] were scaled - where necessary- by Murat Altekin.
} 


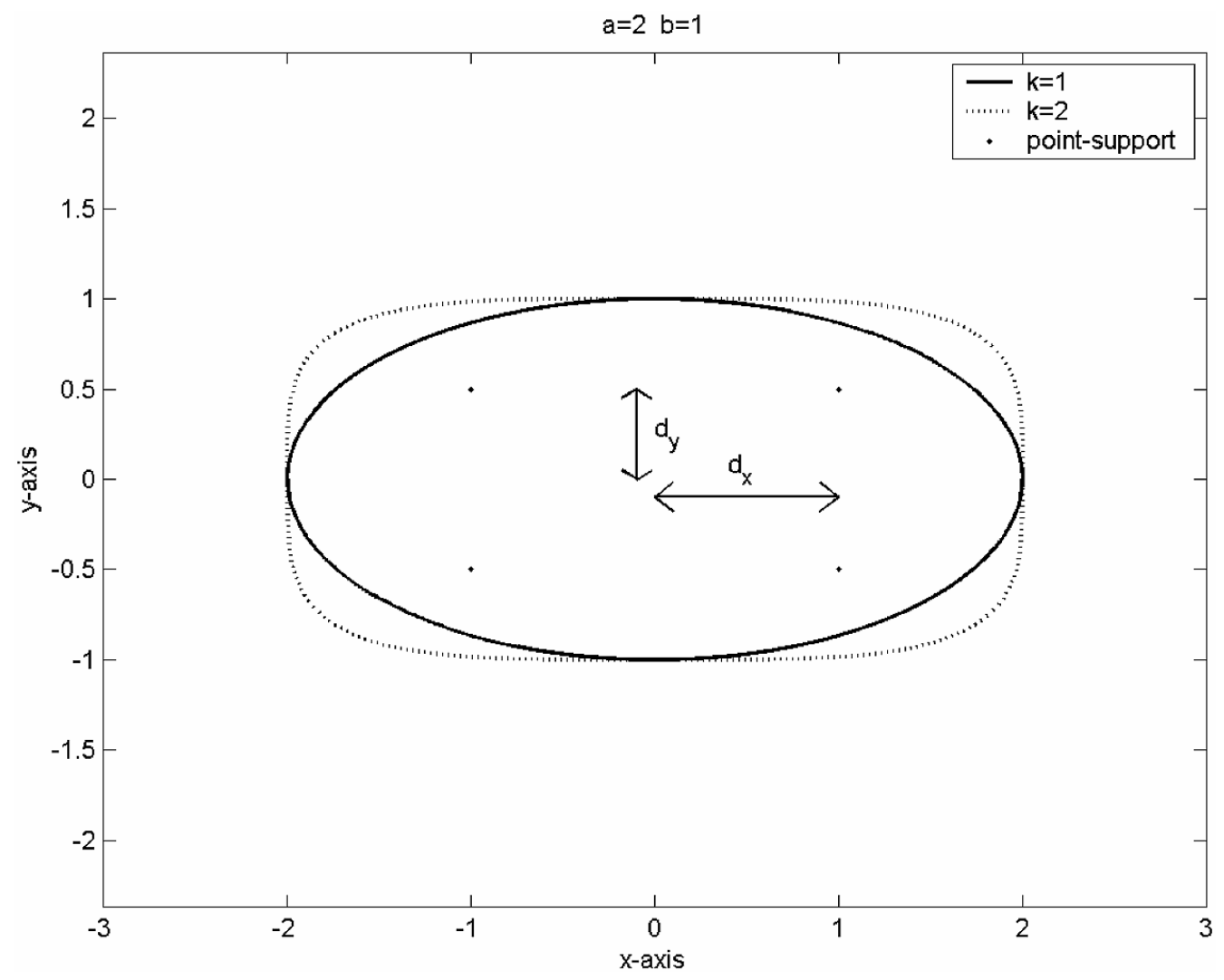

Fig. 1. Geometry of the plate.

\section{Mathematical formulation}

The perimeter of a homogeneous, isotropic, flat, thin super-elliptical elastic plate of uniform thickness $h$ is defined by the equation given by

$$
\left(\frac{x}{a}\right)^{2 k}+\left(\frac{y}{b}\right)^{2 k}=1, \quad k=1,2, \ldots \infty
$$

where $a, b$, and $k$ denote the semi-major axis, the semi-minor axis, and the super-elliptical power respectively [32, $38]$.

The positions of the point-supports are introduced by

$$
d_{x}=\Delta_{1} a, \quad d_{y}=\Delta_{2} b
$$

where $d_{x}$ and $d_{y}$ denote the distance [39] from the central axes of the plate (Fig. 1).

The strain energy $\left(U_{S}\right)$, the kinetic energy $(T)$, and the governing equations $\left(L_{1}, L_{2}\right)$ of the plate are written in terms of $u$ and $v$-the in-plane displacements in $x$-direction and $y$-direction, respectively-, as $[1,6]$

$$
\begin{aligned}
& U_{S}=\frac{D}{2} \int_{x_{1}}^{x_{2}} \int_{y_{1}}^{y_{2}}\left[\left(\frac{\partial u}{\partial x}+\frac{\partial v}{\partial y}\right)^{2}-2(1-\mu) \frac{\partial u}{\partial x} \frac{\partial v}{\partial y}+\frac{1-\mu}{2}\left(\frac{\partial v}{\partial x}+\frac{\partial u}{\partial y}\right)^{2}\right] d x d y \\
& T=\frac{1}{2} \rho h \omega^{2} \int_{x_{1}}^{x_{2}} \int_{y_{1}}^{y_{2}}\left(u^{2}+v^{2}\right) d x d y
\end{aligned}
$$




$$
\begin{aligned}
& L_{1}=C_{11} \frac{\partial^{2} u}{\partial x^{2}}+C_{12} \frac{\partial^{2} v}{\partial x \partial y}+C_{66}\left(\frac{\partial^{2} v}{\partial x \partial y}+\frac{\partial^{2} u}{\partial y^{2}}\right)+\rho u \omega^{2}=0, \\
& L_{2}=C_{66}\left(\frac{\partial^{2} v}{\partial x^{2}}+\frac{\partial^{2} u}{\partial x \partial y}\right)+C_{12} \frac{\partial^{2} u}{\partial x \partial y}+C_{22} \frac{\partial^{2} v}{\partial y^{2}}+\rho v \omega^{2}=0 .
\end{aligned}
$$

Here; $\rho$ denotes the mass density, $\omega$ indicates the frequency, and $\mu$ stands for the Poisson's ratio. The coefficients $\left(C_{11}, C_{12}, C_{22}, C_{66}\right)$ are given by [6]

$$
C_{11}=C_{22}=\frac{E}{\left(1-\mu^{2}\right)}, \quad C_{12}=\frac{\mu E}{\left(1-\mu^{2}\right)}, \quad C_{66}=\frac{E}{2(1+\mu)} .
$$

The extensional rigidity $D$, the aspect ratio $c$, and the non-dimensional counterparts of $x, y, u, v, \omega$ are introduced by

$$
D=\frac{E h}{\left(1-\mu^{2}\right)}, \quad c=\frac{a}{b}, \quad X=\frac{x}{a}, \quad Y=\frac{y}{b}, \quad U=\frac{u}{a}, \quad V=\frac{v}{b}, \quad \lambda^{2}=a^{2} \frac{\rho h}{D} \omega^{2}
$$

where $E$ is the Young's modulus. The plate domain can be characterized by

$$
x_{1}=-a, \quad x_{2}=a, \quad y_{1}=-\frac{1}{c} \sqrt[2 k]{a^{2 k}-x^{2 k}}, \quad y_{2}=\frac{1}{c} \sqrt[2 k]{a^{2 k}-x^{2 k}} .
$$

Due to the symmetry of the geometry of the plate, it is sufficient to consider only a quarter of the plate [1]. Therefore, $U_{S}, T, L_{1}$ and $L_{2}$ can be written as

$$
\begin{aligned}
& U_{S}=\frac{1}{2} a b D \int_{0}^{X_{2}} \int_{0}^{Y_{2}}\left[\left(\frac{\partial U}{\partial X}+\frac{\partial V}{\partial Y}\right)^{2}-2(1-\mu) \frac{\partial U}{\partial X} \frac{\partial V}{\partial Y}+\frac{1-\mu}{2}\left(\frac{1}{c} \frac{\partial V}{\partial X}+c \frac{\partial U}{\partial Y}\right)^{2}\right] d X d Y, \\
& T=\frac{1}{2} a b D \lambda^{2} \int_{0}^{X_{2}} \int_{0}^{Y_{2}}\left(U^{2}+\frac{1}{c^{2}} V^{2}\right) d X d Y \\
& L_{1}=\frac{\partial^{2} U}{\partial X^{2}}+\mu \frac{\partial^{2} V}{\partial X \partial Y}+\frac{(1-\mu)}{2}\left(\frac{\partial^{2} V}{\partial X \partial Y}+c^{2} \frac{\partial^{2} U}{\partial Y^{2}}\right)+U \lambda^{2}=0, \\
& L_{2}=\frac{(1-\mu)}{2}\left(\frac{1}{c} \frac{\partial^{2} V}{\partial X^{2}}+c \frac{\partial^{2} U}{\partial X \partial Y}\right)+\mu c \frac{\partial^{2} U}{\partial X \partial Y}+c \frac{\partial^{2} V}{\partial Y^{2}}+\frac{1}{c} V \lambda^{2}=0
\end{aligned}
$$

where

$$
X_{2}=1, \quad Y_{2}=\sqrt[2 k]{1-X^{2 k}}
$$

To improve the accuracy of the results the total residual error is set to zero by

$$
G_{1}=\int_{0}^{X_{2}} \int_{0}^{Y_{2}} L_{1} d X d Y=0, \quad G_{2}=\int_{0}^{X_{2}} \int_{0}^{Y_{2}} L_{2} d X d Y=0
$$

The shape functions $U$ and $V$ are complete two-dimensional polynomial functions of degree $d$ defined by [1]

$$
U=\sum_{m=0}^{M} \sum_{n=0}^{N} A_{m n} X^{m} Y^{n}, \quad V=\sum_{m=0}^{M} \sum_{n=0}^{N} B_{m n} X^{m} Y^{n}, \quad d=m+n .
$$

Here; $A_{m n}$ and $B_{m n}$ are the unknown coefficients. Depending on the mode category, the shape functions are constructed (Table 1). Since the in-plane displacement is prevented at the point-supports, the subsidiary conditions [40] can be written as

$$
G_{3}=U\left(X=\Delta_{1}, Y=\Delta_{2}\right), \quad G_{4}=V\left(X=\Delta_{1}, Y=\Delta_{2}\right) .
$$


Table 1

Construction of the shape functions [1]

\begin{tabular}{cccccc}
\hline & \multicolumn{2}{c}{$U$} & & \multicolumn{2}{c}{$V$} \\
\cline { 2 - 3 } \cline { 5 - 6 } Mode & $m$ & $n$ & & $m$ & $n$ \\
\hline SS & odd & even & & even & odd \\
SA & even & even & & odd & odd \\
AS & odd & odd & & even & even \\
AA & even & odd & & odd & even \\
\hline
\end{tabular}

Table 2

Convergence study for $\lambda(k=200)$

\begin{tabular}{|c|c|c|c|c|c|c|c|c|c|c|c|}
\hline \multirow[b]{2}{*}{$c$} & \multirow[b]{2}{*}{ Mode } & \multirow[b]{2}{*}{$d$} & \multicolumn{3}{|c|}{ Case 1} & \multicolumn{3}{|c|}{ Case $2(\Delta=0.5)$} & \multicolumn{3}{|c|}{ Case $2(\Delta=0.9)$} \\
\hline & & & Mode-1 & Mode-2 & Mode-3 & Mode-1 & Mode-2 & Mode-3 & Mode-1 & Mode-2 & Mode-3 \\
\hline \multirow[t]{3}{*}{1} & SS & 11 & 1.3143 & 1.4938 & 1.7263 & 1.4890 & 2.0687 & 2.2726 & 1.3168 & 2.0956 & 2.4490 \\
\hline & & 13 & 1.3143 & 1.4938 & 1.7263 & 1.4888 & 2.0177 & 2.2424 & 1.3168 & 2.0858 & 2.4313 \\
\hline & & 15 & 1.3143 & 1.4938 & 1.7263 & 1.4886 & 1.9538 & 2.2151 & 1.3168 & 2.0763 & 2.4139 \\
\hline \multirow[t]{3}{*}{1} & SA & 10 & 1.2359 & 1.8616 & 2.4857 & 1.3253 & 1.7635 & 2.4463 & 0.7099 & 1.8296 & 2.2346 \\
\hline & & 12 & 1.2359 & 1.8616 & 2.4845 & 1.3052 & 1.5973 & 2.3834 & 0.6943 & 1.8188 & 2.2160 \\
\hline & & 14 & 1.2359 & 1.8616 & 2.4844 & 1.2996 & 1.5574 & 2.3616 & 0.6808 & 1.8079 & 2.2000 \\
\hline \multirow[t]{3}{*}{1} & AA & 11 & 1.1604 & 2.1516 & 2.6285 & 1.1713 & 2.0554 & 2.6285 & 1.0392 & 1.7270 & 2.5920 \\
\hline & & 13 & 1.1604 & 2.1516 & 2.6285 & 1.1395 & 2.0118 & 2.6285 & 1.0247 & 1.6941 & 2.5904 \\
\hline & & 15 & 1.1604 & 2.1516 & 2.6285 & 1.0918 & 1.9614 & 2.6285 & 1.0114 & 1.6762 & 2.5888 \\
\hline \multirow[t]{3}{*}{2} & SS & 11 & 1.4805 & 2.6023 & 3.0734 & 2.5228 & 2.8713 & 3.2863 & 1.8798 & 3.1506 & 3.4352 \\
\hline & & 13 & 1.4805 & 2.6023 & 3.0734 & 2.4909 & 2.8017 & 3.2857 & 1.8668 & 3.1475 & 3.4183 \\
\hline & & 15 & 1.4805 & 2.6023 & 3.0734 & 2.4454 & 2.7448 & 3.2850 & 1.8590 & 3.1448 & 3.4079 \\
\hline \multirow[t]{3}{*}{2} & SA & 10 & 2.6285 & 2.6827 & 3.3746 & 2.5030 & 2.8361 & 3.0762 & 1.0288 & 2.6333 & 3.4964 \\
\hline & & 12 & 2.6285 & 2.6826 & 3.3746 & 2.2686 & 2.7389 & 3.0387 & 1.0002 & 2.6332 & 3.4911 \\
\hline & & 14 & 2.6285 & 2.6826 & 3.3746 & 2.2083 & 2.7293 & 3.0302 & 0.9826 & 2.6331 & 3.4865 \\
\hline \multirow[t]{3}{*}{2} & AS & 10 & 0.9770 & 2.3922 & 3.2285 & 1.3042 & 1.7665 & 3.1676 & 0.8118 & 2.2264 & 3.2996 \\
\hline & & 12 & 0.9770 & 2.3921 & 3.2240 & 1.2558 & 1.6888 & 3.0556 & 0.7972 & 2.2012 & 3.2640 \\
\hline & & 14 & 0.9770 & 2.3921 & 3.2238 & 1.2440 & 1.6615 & 3.0047 & 0.7817 & 2.1591 & 3.2346 \\
\hline \multirow[t]{3}{*}{2} & AA & 11 & 1.6336 & 2.3633 & 3.4289 & 1.3474 & 2.9460 & 3.2347 & 1.4300 & 2.4166 & 3.1466 \\
\hline & & 13 & 1.6336 & 2.3633 & 3.4286 & 1.3195 & 2.8818 & 3.1876 & 1.4068 & 2.3987 & 3.0983 \\
\hline & & 15 & 1.6336 & 2.3633 & 3.4286 & 1.2781 & 2.8126 & 3.1189 & 1.3818 & 2.3831 & 3.0539 \\
\hline
\end{tabular}

Consequently, the modified total potential energy functional of the plate becomes

$$
F=U_{S}-T+\beta_{1} G_{1}+\beta_{2} G_{2}+\eta\left(\beta_{3} G_{3}+\beta_{4} G_{4}\right)
$$

where $\beta_{1}, \beta_{2}, \beta_{3}, \beta_{4}$ are the Lagrange multipliers. The scalar indicator [35] $\eta$ is designated as follows:

- $\eta=0$ for a completely free plate (Case 1$)$,

- $\eta=1$ for a point-supported plate (Case 2).

The Ritz method is applied by

$$
\frac{\partial F}{\partial A_{m n}}=0, \quad \frac{\partial F}{\partial B_{m n}}=0, \quad \frac{\partial F}{\partial \beta_{1}}=0, \quad \frac{\partial F}{\partial \beta_{2}}=0, \quad \frac{\partial F}{\partial \beta_{3}}=0, \quad \frac{\partial F}{\partial \beta_{4}}=0 .
$$

The formulation becomes

$$
\left[K_{3}\right]=\left[K_{1}\right]-\lambda^{2}\left[K_{2}\right], \quad\left[K_{3}\right]\{s\}=\{0\}
$$

where $\{s\}$ is the vector composed of the unknowns $A_{m n}, B_{m n}, \beta_{1}, \beta_{2}, \beta_{3}, \beta_{4}$. The square matrices $\left[K_{1}\right]$, and $\left[K_{2}\right]$ are obtained by using Eq. (19). For a non-trivial solution, the determinant of the coefficient matrix $\left[K_{3}\right]$ is set to zero to obtain $\lambda$.

\section{Numerical results}

The integration with respect to " $X$ " was evaluated by the 12-point Gaussian quadrature technique. The free in-plane vibration modes were classified into SS, SA, AS, and AA groups. The convergence studies reveal that a 
Table 3

Frequency parameter $\lambda($ Case $1, c=1)$

\begin{tabular}{|c|c|c|c|c|c|c|c|c|c|}
\hline$c=1$ & \multicolumn{3}{|c|}{$d=15$} & \multicolumn{3}{|c|}{$d=14$} & \multicolumn{3}{|c|}{$d=15$} \\
\hline$k$ & SS-1 & SS-2 & SS-3 & SA-1 & SA-2 & SA-3 & AA-1 & AA-2 & AA-3 \\
\hline 1 & 1.3892 & 2.0500 & 2.5125 & 1.6177 & 2.1303 & 3.3771 & 1.3894 & 2.5115 & 2.7749 \\
\hline 2 & 1.3261 & 1.8522 & 1.8617 & 1.4078 & 1.9592 & 2.8991 & 1.2351 & 2.5062 & 2.6493 \\
\hline 3 & 1.3178 & 1.6880 & 1.7932 & 1.3302 & 1.9161 & 2.7264 & 1.1981 & 2.3857 & 2.6543 \\
\hline 4 & 1.3157 & 1.6151 & 1.7675 & 1.2954 & 1.8970 & 2.6458 & 1.1832 & 2.3141 & 2.6402 \\
\hline 5 & 1.3149 & 1.5770 & 1.7541 & 1.2769 & 1.8865 & 2.6003 & 1.1756 & 2.2701 & 2.6338 \\
\hline 6 & 1.3147 & 1.5545 & 1.7463 & 1.2657 & 1.8800 & 2.5718 & 1.1713 & 2.2417 & 2.6313 \\
\hline 7 & 1.3145 & 1.5400 & 1.7413 & 1.2586 & 1.8758 & 2.5525 & 1.1685 & 2.2222 & 2.6300 \\
\hline 8 & 1.3143 & 1.5302 & 1.7380 & 1.2538 & 1.8728 & 2.5390 & 1.1666 & 2.2084 & 2.6294 \\
\hline 9 & 1.3143 & 1.5232 & 1.7357 & 1.2504 & 1.8707 & 2.5291 & 1.1653 & 2.1982 & 2.6291 \\
\hline 10 & 1.3143 & 1.5179 & 1.7339 & 1.2478 & 1.8692 & 2.5217 & 1.1645 & 2.1904 & 2.6289 \\
\hline 11 & 1.3143 & 1.5139 & 1.7326 & 1.2458 & 1.8679 & 2.5159 & 1.1638 & 2.1845 & 2.6288 \\
\hline 12 & 1.3143 & 1.5110 & 1.7316 & 1.2444 & 1.8669 & 2.5114 & 1.1632 & 2.1798 & 2.6287 \\
\hline 13 & 1.3143 & 1.5085 & 1.7308 & 1.2432 & 1.8663 & 2.5077 & 1.1628 & 2.1760 & 2.6286 \\
\hline 14 & 1.3143 & 1.5065 & 1.7302 & 1.2422 & 1.8656 & 2.5047 & 1.1625 & 2.1729 & 2.6286 \\
\hline 15 & 1.3143 & 1.5050 & 1.7296 & 1.2414 & 1.8652 & 2.5023 & 1.1621 & 2.1704 & 2.6286 \\
\hline 16 & 1.3143 & 1.5037 & 1.7292 & 1.2408 & 1.8647 & 2.5002 & 1.1619 & 2.1682 & 2.6286 \\
\hline 17 & 1.3143 & 1.5025 & 1.7289 & 1.2402 & 1.8644 & 2.4985 & 1.1619 & 2.1663 & 2.6285 \\
\hline 18 & 1.3143 & 1.5015 & 1.7286 & 1.2398 & 1.8641 & 2.4970 & 1.1617 & 2.1648 & 2.6285 \\
\hline 19 & 1.3143 & 1.5008 & 1.7283 & 1.2394 & 1.8639 & 2.4958 & 1.1615 & 2.1636 & 2.6285 \\
\hline 20 & 1.3143 & 1.5002 & 1.7281 & 1.2390 & 1.8636 & 2.4947 & 1.1615 & 2.1624 & 2.6285 \\
\hline 50 & 1.3143 & 1.4947 & 1.7264 & 1.2363 & 1.8619 & 2.4859 & 1.1606 & 2.1531 & 2.6285 \\
\hline 200 & 1.3143 & 1.4938 & 1.7263 & 1.2359 & 1.8616 & 2.4844 & 1.1604 & 2.1516 & 2.6285 \\
\hline \multicolumn{10}{|l|}{ Ref. } \\
\hline$[1]$ & 1.3140 & 1.4940 & 1.7260 & & & & 1.1600 & & \\
\hline [2] & 1.3140 & 1.4935 & 1.7260 & 1.2360 & & & 1.1605 & & \\
\hline
\end{tabular}

Table 4

Frequency parameter $\lambda($ Case $1, c=2)$

\begin{tabular}{|c|c|c|c|c|c|c|c|c|c|c|c|c|}
\hline \multirow{2}{*}{$\frac{c=2}{k}$} & \multicolumn{3}{|c|}{$d=15$} & \multicolumn{3}{|c|}{$d=14$} & \multicolumn{3}{|c|}{$d=14$} & \multicolumn{3}{|c|}{$d=15$} \\
\hline & SS-1 & SS-2 & SS-3 & SA-1 & SA-2 & SA-3 & AS-1 & AS-2 & AS-3 & $\mathrm{AA}-1$ & AA-2 & AA-3 \\
\hline 1 & 1.7101 & 3.2295 & 3.6598 & 2.8418 & 3.4679 & 4.1233 & 1.2538 & 2.8283 & 3.7467 & 1.9836 & 2.6012 & 3.6831 \\
\hline 2 & 1.5601 & 3.0004 & 3.2599 & 2.6700 & 3.3060 & 3.5942 & 1.0890 & 2.5978 & 3.4461 & 1.7779 & 2.4893 & 3.5781 \\
\hline 3 & 1.5209 & 2.8705 & 3.1650 & 2.6415 & 3.0707 & 3.4845 & 1.0368 & 2.5095 & 3.3624 & 1.7133 & 2.4361 & 3.5249 \\
\hline 4 & 1.5048 & 2.7879 & 3.1270 & 2.6338 & 2.9372 & 3.4475 & 1.0141 & 2.4678 & 3.3200 & 1.6842 & 2.4105 & 3.4954 \\
\hline 5 & 1.4967 & 2.7367 & 3.1084 & 2.6311 & 2.8619 & 3.4270 & 1.0022 & 2.4448 & 3.2946 & 1.6685 & 2.3962 & 3.4775 \\
\hline 6 & 1.4920 & 2.7036 & 3.0980 & 2.6299 & 2.8158 & 3.4139 & 0.9952 & 2.4308 & 3.2781 & 1.6591 & 2.3876 & 3.4658 \\
\hline 7 & 1.4891 & 2.6812 & 3.0915 & 2.6294 & 2.7853 & 3.4051 & 0.9910 & 2.4217 & 3.2667 & 1.6530 & 2.3819 & 3.4578 \\
\hline 8 & 1.4871 & 2.6654 & 3.0873 & 2.6290 & 2.7642 & 3.3989 & 0.9879 & 2.4154 & 3.2585 & 1.6489 & 2.3779 & 3.4520 \\
\hline 9 & 1.4858 & 2.6539 & 3.0844 & 2.6289 & 2.7490 & 3.3944 & 0.9856 & 2.4109 & 3.2524 & 1.6459 & 2.3752 & 3.4478 \\
\hline 10 & 1.4848 & 2.6452 & 3.0823 & 2.6287 & 2.7376 & 3.3909 & 0.9841 & 2.4076 & 3.2478 & 1.6438 & 2.3731 & 3.4446 \\
\hline 11 & 1.4841 & 2.6385 & 3.0807 & 2.6287 & 2.7288 & 3.3884 & 0.9829 & 2.4050 & 3.2441 & 1.6421 & 2.3715 & 3.4421 \\
\hline 12 & 1.4836 & 2.6332 & 3.0795 & 2.6286 & 2.7220 & 3.3863 & 0.9821 & 2.4030 & 3.2413 & 1.6407 & 2.3702 & 3.4401 \\
\hline 13 & 1.4831 & 2.6290 & 3.0786 & 2.6286 & 2.7166 & 3.3847 & 0.9813 & 2.4015 & 3.2390 & 1.6398 & 2.3692 & 3.4385 \\
\hline 14 & 1.4827 & 2.6255 & 3.0778 & 2.6286 & 2.7122 & 3.3833 & 0.9808 & 2.4002 & 3.2371 & 1.6389 & 2.3684 & 3.4372 \\
\hline 15 & 1.4824 & 2.6227 & 3.0773 & 2.6286 & 2.7086 & 3.3822 & 0.9803 & 2.3992 & 3.2355 & 1.6383 & 2.3678 & 3.4361 \\
\hline 16 & 1.4822 & 2.6203 & 3.0768 & 2.6285 & 2.7055 & 3.3813 & 0.9798 & 2.3983 & 3.2342 & 1.6377 & 2.3673 & 3.4353 \\
\hline 17 & 1.4821 & 2.6183 & 3.0764 & 2.6285 & 2.7031 & 3.3806 & 0.9795 & 2.3976 & 3.2330 & 1.6372 & 2.3667 & 3.4345 \\
\hline 18 & 1.4819 & 2.6167 & 3.0760 & 2.6285 & 2.7008 & 3.3799 & 0.9793 & 2.3971 & 3.2321 & 1.6369 & 2.3664 & 3.4339 \\
\hline 19 & 1.4817 & 2.6152 & 3.0757 & 2.6285 & 2.6991 & 3.3794 & 0.9790 & 2.3966 & 3.2313 & 1.6365 & 2.3661 & 3.4334 \\
\hline 20 & 1.4816 & 2.6140 & 3.0755 & 2.6285 & 2.6975 & 3.3790 & 0.9788 & 2.3961 & 3.2306 & 1.6362 & 2.3658 & 3.4329 \\
\hline 50 & 1.4807 & 2.6039 & 3.0736 & 2.6285 & 2.6848 & 3.3752 & 0.9772 & 2.3927 & 3.2248 & 1.6340 & 2.3636 & 3.4291 \\
\hline 200 & 1.4805 & 2.6023 & 3.0734 & 2.6285 & 2.6826 & 3.3746 & 0.9770 & 2.3921 & 3.2238 & 1.6336 & 2.3633 & 3.4286 \\
\hline \multicolumn{13}{|l|}{ Ref. } \\
\hline [1] & 1.4807 & 2.6022 & & & & & 0.9768 & 2.3914 & & 1.6334 & 2.3632 & \\
\hline [2] & 1.4805 & 2.6025 & & & & & 0.9770 & 2.3920 & & 1.6335 & 2.3630 & \\
\hline
\end{tabular}


Table 5

Comparison of $\lambda$ for the first six modes (Case $1, k=200$ )

\begin{tabular}{lllllll}
\hline & \multicolumn{7}{c}{ Mode number } \\
\cline { 3 - 7 }$c$ & 1 & 2 & 3 & 4 & 5 & 6 \\
\hline 1 & 1.1604 & 1.2359 & 1.2359 & 1.3143 & 1.4938 & 1.7263 \\
& $1.1605[2]$ & $1.2360[2]$ & $1.2360[2]$ & $1.3140[2]$ & $1.4935[2]$ & $1.7260[2]$ \\
& $1.1505[3]$ & $1.2355[3]$ & $1.2355[3]$ & $1.2945[3]$ & $1.4855[3]$ & $1.7505[3]$ \\
& $1.1605[4]$ & $1.2360[4]$ & $1.2360[4]$ & $1.3145[4]$ & $1.4940[4]$ & $1.7260[4]$ \\
& $1.1600[5]$ & $1.2360[5]$ & $1.2360[5]$ & $1.3140[5]$ & $1.4940[5]$ & $1.7260[5]$ \\
& $1.1600[6]$ & $1.2360[6]$ & $1.2360[6]$ & $1.3140[6]$ & $1.4940[6]$ & $1.7260[6]$ \\
2 & 0.9770 & 1.4805 & 1.6336 & 2.3633 & 2.3921 & 2.6023 \\
& $0.9768[1]$ & $1.4807[1]$ & $1.6334[1]$ & $2.3632[1]$ & $2.3914[1]$ & $2.6022[1]$ \\
& $0.9770[2]$ & $1.4805[2]$ & $1.6350[2]$ & $2.3630[2]$ & $2.3920[2]$ & $2.6025[2]$ \\
& $0.9690[3]$ & $1.4635[3]$ & $1.6190[3]$ & $2.3510[3]$ & $2.3760[3]$ & $2.5890[3]$ \\
& $0.9770[4]$ & $1.4805[4]$ & $1.6340[4]$ & $2.3625[4]$ & $2.3925[4]$ & $2.6025[4]$ \\
3 & 0.7855 & 1.4916 & 1.6112 & 2.4740 & 2.8758 & 2.9149 \\
& $0.7855[4]$ & $1.4915[4]$ & $1.6120[4]$ & $2.4755[4]$ & $2.8770[4]$ & $2.9150[4]$ \\
\hline
\end{tabular}

Table 6

Frequency parameter $\lambda$ (Case $2, c=1, d=15, \mathrm{SS}-1)$

\begin{tabular}{|c|c|c|c|c|c|c|c|c|c|}
\hline$k$ & $\Delta=0.1$ & $\Delta=0.2$ & $\Delta=0.3$ & $\Delta=0.4$ & $\Delta=0.5$ & $\Delta=0.6$ & $\Delta=0.7$ & $\Delta=0.8$ & $\Delta=0.9$ \\
\hline 1 & 1.5246 & 1.7696 & 1.9707 & 2.0653 & 1.8130 & 1.5734 & 1.4274 & - & - \\
\hline 2 & 1.4353 & 1.5870 & 1.7032 & 1.8538 & 1.7238 & 1.5269 & 1.4023 & 1.3429 & - \\
\hline 3 & 1.4209 & 1.5346 & 1.6061 & 1.6790 & 1.6439 & 1.4983 & 1.3907 & 1.3372 & - \\
\hline 4 & 1.4147 & 1.5053 & 1.5568 & 1.6065 & 1.5928 & 1.4804 & 1.3842 & 1.3351 & 1.3180 \\
\hline 5 & 1.4112 & 1.4878 & 1.5294 & 1.5691 & 1.5624 & 1.4685 & 1.3799 & 1.3338 & 1.3174 \\
\hline 6 & 1.4089 & 1.4767 & 1.5126 & 1.5469 & 1.5434 & 1.4605 & 1.3771 & 1.3330 & 1.3172 \\
\hline 7 & 1.4073 & 1.4692 & 1.5018 & 1.5328 & 1.5307 & 1.4550 & 1.3750 & 1.3325 & 1.3172 \\
\hline 8 & 1.4061 & 1.4639 & 1.4943 & 1.5232 & 1.5220 & 1.4509 & 1.3735 & 1.3321 & 1.3172 \\
\hline 9 & 1.4052 & 1.4601 & 1.4890 & 1.5164 & 1.5158 & 1.4479 & 1.3724 & 1.3317 & 1.3170 \\
\hline 10 & 1.4046 & 1.4574 & 1.4849 & 1.5113 & 1.5110 & 1.4457 & 1.3717 & 1.3315 & 1.3170 \\
\hline 11 & 1.4041 & 1.4552 & 1.4821 & 1.5075 & 1.5073 & 1.4438 & 1.3709 & 1.3314 & 1.3170 \\
\hline 12 & 1.4036 & 1.4534 & 1.4797 & 1.5045 & 1.5045 & 1.4424 & 1.3704 & 1.3312 & 1.3170 \\
\hline 13 & 1.4034 & 1.4522 & 1.4778 & 1.5022 & 1.5023 & 1.4414 & 1.3700 & 1.3310 & 1.3170 \\
\hline 14 & 1.4030 & 1.4510 & 1.4763 & 1.5003 & 1.5005 & 1.4405 & 1.3697 & 1.3310 & 1.3170 \\
\hline 15 & 1.4029 & 1.4502 & 1.4751 & 1.4988 & 1.4990 & 1.4396 & 1.3693 & 1.3308 & 1.3170 \\
\hline 16 & 1.4027 & 1.4495 & 1.4741 & 1.4975 & 1.4978 & 1.4391 & 1.3691 & 1.3308 & 1.3170 \\
\hline 17 & 1.4025 & 1.4490 & 1.4733 & 1.4965 & 1.4968 & 1.4384 & 1.3689 & 1.3308 & 1.3170 \\
\hline 18 & 1.4025 & 1.4484 & 1.4726 & 1.4955 & 1.4960 & 1.4381 & 1.3688 & 1.3308 & 1.3170 \\
\hline 19 & 1.4023 & 1.4479 & 1.4719 & 1.4948 & 1.4952 & 1.4377 & 1.3686 & 1.3306 & 1.3170 \\
\hline 20 & 1.4023 & 1.4476 & 1.4714 & 1.4942 & 1.4947 & 1.4374 & 1.3684 & 1.3306 & 1.3168 \\
\hline 50 & 1.4018 & 1.4445 & 1.4673 & 1.4890 & 1.4895 & 1.4346 & 1.3675 & 1.3302 & 1.3168 \\
\hline 200 & 1.4016 & 1.4441 & 1.4668 & 1.4881 & 1.4886 & 1.4342 & 1.3673 & 1.3302 & 1.3168 \\
\hline $\begin{array}{c}\text { Ref. } \\
\text { [1] }\end{array}$ & & & & & 1.4880 & & & & 1.3170 \\
\hline
\end{tabular}

polynomial function of degree $d=15$ (for SS and AA modes), and $d=14$ (for SA and AS modes) is sufficient for admissible accuracy (Table 2).

The first three in-plane free vibration frequencies of either completely free (Tables 3-5) or point-supported superelliptical plates were carried out numerically for four mode categories for the parameters shown in the following:

$$
k=\{1,2, \ldots 20,50,200\}, \quad c=\{1,2\}, \quad \mu=0.3, \quad \Delta=\Delta_{1}=\Delta_{2}, \quad 0.1 \leqslant \Delta \leqslant 0.9
$$

To avoid ambiguity of excessive data, some of the results were presented for the second case (Tables 6-13).

\section{Concluding remarks}

The in-plane free vibration frequencies corresponding to the first three SS, SA, AS, and AA modes of isotropic super-elliptical plates of uniform thickness were investigated by the Ritz method. Various plate shapes for two 
Table 7

Frequency parameter $\lambda$ (Case 2, $c=1, d=14, \mathrm{SA}-1)$

\begin{tabular}{ccccccccccc}
\hline$k$ & $\Delta=0.1$ & $\Delta=0.2$ & $\Delta=0.3$ & $\Delta=0.4$ & $\Delta=0.5$ & $\Delta=0.6$ & $\Delta=0.7$ & $\Delta=0.8$ & $\Delta=0.9$ \\
\hline 1 & 0.8698 & 1.0766 & 1.2839 & 1.5547 & 1.4753 & 1.1679 & 0.8755 & - & - \\
2 & 0.7714 & 0.9341 & 1.0954 & 1.2917 & 1.5158 & 1.2311 & 0.9962 & 0.7864 & - \\
3 & 0.7457 & 0.8950 & 1.0387 & 1.2122 & 1.4381 & 1.2367 & 1.0164 & 0.8204 & - \\
4 & 0.7352 & 0.8789 & 1.0141 & 1.1783 & 1.3878 & 1.2381 & 1.0247 & 0.8355 & 0.6438 \\
5 & 0.7301 & 0.8709 & 1.0015 & 1.1608 & 1.3605 & 1.2390 & 1.0291 & 0.8429 & 0.6584 \\
6 & 0.7270 & 0.8663 & 0.9942 & 1.1507 & 1.3442 & 1.2396 & 1.0315 & 0.8468 & 0.6671 \\
7 & 0.7253 & 0.8634 & 0.9897 & 1.1443 & 1.3338 & 1.2400 & 1.0327 & 0.8488 & 0.6719 \\
8 & 0.7242 & 0.8614 & 0.9864 & 1.1400 & 1.3265 & 1.2404 & 1.0334 & 0.8500 & 0.6749 \\
9 & 0.7232 & 0.8599 & 0.9841 & 1.1369 & 1.3214 & 1.2406 & 1.0339 & 0.8509 & 0.6768 \\
10 & 0.7228 & 0.8591 & 0.9826 & 1.1347 & 1.3176 & 1.2408 & 1.0342 & 0.8512 & 0.6779 \\
11 & 0.7225 & 0.8582 & 0.9813 & 1.1329 & 1.3145 & 1.2412 & 1.0344 & 0.8515 & 0.6786 \\
12 & 0.7221 & 0.8579 & 0.9803 & 1.1316 & 1.3122 & 1.2414 & 1.0346 & 0.8515 & 0.6793 \\
13 & 0.7218 & 0.8573 & 0.9795 & 1.1307 & 1.3105 & 1.2416 & 1.0346 & 0.8518 & 0.6797 \\
14 & 0.7215 & 0.8570 & 0.9790 & 1.1298 & 1.3090 & 1.2416 & 1.0349 & 0.8518 & 0.6797 \\
15 & 0.7215 & 0.8567 & 0.9785 & 1.1292 & 1.3079 & 1.2418 & 1.0349 & 0.8518 & 0.6801 \\
16 & 0.7215 & 0.8564 & 0.9780 & 1.1285 & 1.3069 & 1.2420 & 1.0349 & 0.8518 & 0.6801 \\
17 & 0.7211 & 0.8564 & 0.9778 & 1.1281 & 1.3061 & 1.2420 & 1.0351 & 0.8521 & 0.6804 \\
18 & 0.7211 & 0.8562 & 0.9772 & 1.1278 & 1.3054 & 1.2422 & 1.0351 & 0.8521 & 0.6804 \\
19 & 0.7211 & 0.8562 & 0.9770 & 1.1274 & 1.3048 & 1.2424 & 1.0351 & 0.8521 & 0.6804 \\
20 & 0.7211 & 0.8559 & 0.9770 & 1.1272 & 1.3044 & 1.2424 & 1.0351 & 0.8521 & 0.6804 \\
50 & 0.7208 & 0.8550 & 0.9752 & 1.1252 & 1.3004 & 1.2436 & 1.0354 & 0.8521 & 0.6804 \\
200 & 0.7208 & 0.8550 & 0.9749 & 1.1249 & 1.2996 & 1.2440 & 1.0356 & 0.8521 & 0.6808
\end{tabular}

Ref.

Table 8

Frequency parameter $\lambda$ (Case 2, $c=1, d=15$, AA-1)

\begin{tabular}{ccccccccccc}
\hline$k$ & $\Delta=0.1$ & $\Delta=0.2$ & $\Delta=0.3$ & $\Delta=0.4$ & $\Delta=0.5$ & $\Delta=0.6$ & $\Delta=0.7$ & $\Delta=0.8$ & $\Delta=0.9$ \\
\hline 1 & 0.3225 & 0.5688 & 0.8772 & 1.2085 & 1.6206 & 1.7563 & 1.3387 & - & - \\
2 & 0.2608 & 0.4588 & 0.6845 & 0.9703 & 1.2744 & 1.5435 & 1.5897 & 1.2871 & - \\
3 & 0.2540 & 0.4387 & 0.6515 & 0.9203 & 1.1889 & 1.4607 & 1.5973 & 1.3396 & - \\
4 & 0.2520 & 0.4307 & 0.6395 & 0.8989 & 1.1502 & 1.4271 & 1.5992 & 1.3605 & 0.9612 \\
5 & 0.2510 & 0.4266 & 0.6340 & 0.8874 & 1.1300 & 1.4093 & 1.5969 & 1.3671 & 0.9907 \\
6 & 0.2510 & 0.4243 & 0.6309 & 0.8803 & 1.1185 & 1.3984 & 1.5930 & 1.3697 & 1.0025 \\
7 & 0.2510 & 0.4225 & 0.6289 & 0.8758 & 1.1113 & 1.3914 & 1.5889 & 1.3706 & 1.0072 \\
8 & 0.2510 & 0.4219 & 0.6277 & 0.8726 & 1.1066 & 1.3867 & 1.5856 & 1.3711 & 1.0087 \\
9 & 0.2510 & 0.4207 & 0.6269 & 0.8706 & 1.1034 & 1.3833 & 1.5829 & 1.3715 & 1.0092 \\
10 & 0.2510 & 0.4207 & 0.6265 & 0.8689 & 1.1009 & 1.3809 & 1.5807 & 1.3717 & 1.0095 \\
11 & 0.2510 & 0.4201 & 0.6261 & 0.8678 & 1.0991 & 1.3791 & 1.5788 & 1.3719 & 1.0097 \\
12 & 0.2510 & 0.4201 & 0.6261 & 0.8669 & 1.0977 & 1.3779 & 1.5773 & 1.3720 & 1.0100 \\
13 & 0.2510 & 0.4195 & 0.6257 & 0.8660 & 1.0968 & 1.3768 & 1.5762 & 1.3720 & 1.0102 \\
14 & 0.2510 & 0.4195 & 0.6257 & 0.8654 & 1.0959 & 1.3759 & 1.5753 & 1.3722 & 1.0102 \\
15 & 0.2510 & 0.4195 & 0.6257 & 0.8649 & 1.0952 & 1.3753 & 1.5743 & 1.3724 & 1.0104 \\
16 & 0.2510 & 0.4195 & 0.6257 & 0.8646 & 1.0948 & 1.3748 & 1.5737 & 1.3724 & 1.0104 \\
17 & 0.2510 & 0.4189 & 0.6257 & 0.8643 & 1.0943 & 1.3742 & 1.5731 & 1.3726 & 1.0104 \\
18 & 0.2510 & 0.4189 & 0.6257 & 0.8640 & 1.0941 & 1.3739 & 1.5726 & 1.3726 & 1.0107 \\
19 & 0.2510 & 0.4189 & 0.6257 & 0.8637 & 1.0936 & 1.3735 & 1.5721 & 1.3728 & 1.0107 \\
20 & 0.2520 & 0.4189 & 0.6257 & 0.8634 & 1.0934 & 1.3733 & 1.5718 & 1.3728 & 1.0107 \\
50 & 0.2520 & 0.4183 & 0.6257 & 0.8617 & 1.0920 & 1.3715 & 1.5686 & 1.3739 & 1.0112 \\
200 & 0.2520 & 0.4183 & 0.6257 & 0.8614 & 1.0918 & 1.3713 & 1.5681 & 1.3740 & 1.0114
\end{tabular}

Ref. 
Table 9

Frequency parameter $\lambda($ Case $2, c=2, d=15$, SS-1)

\begin{tabular}{|c|c|c|c|c|c|c|c|c|c|}
\hline$k$ & $\Delta=0.1$ & $\Delta=0.2$ & $\Delta=0.3$ & $\Delta=0.4$ & $\Delta=0.5$ & $\Delta=0.6$ & $\Delta=0.7$ & $\Delta=0.8$ & $\Delta=0.9$ \\
\hline 1 & 1.8319 & 2.0305 & 2.3103 & 2.6615 & 2.8497 & 2.5846 & 2.2139 & - & - \\
\hline 2 & 1.6488 & 1.7909 & 1.9940 & 2.3170 & 2.6709 & 2.5617 & 2.3042 & 2.0277 & - \\
\hline 3 & 1.6069 & 1.7381 & 1.9289 & 2.2345 & 2.5937 & 2.5498 & 2.2982 & 2.0408 & - \\
\hline 4 & 1.5903 & 1.7168 & 1.9037 & 2.1980 & 2.5472 & 2.5465 & 2.2933 & 2.0457 & 1.8321 \\
\hline 5 & 1.5821 & 1.7062 & 1.8913 & 2.1783 & 2.5187 & 2.5434 & 2.2888 & 2.0466 & 1.8442 \\
\hline 6 & 1.5775 & 1.7001 & 1.8843 & 2.1661 & 2.5002 & 2.5404 & 2.2851 & 2.0462 & 1.8507 \\
\hline 7 & 1.5746 & 1.6963 & 1.8800 & 2.1581 & 2.4876 & 2.5375 & 2.2821 & 2.0456 & 1.8539 \\
\hline 8 & 1.5729 & 1.6937 & 1.8772 & 2.1526 & 2.4786 & 2.5351 & 2.2798 & 2.0450 & 1.8557 \\
\hline 9 & 1.5716 & 1.6919 & 1.8752 & 2.1486 & 2.4720 & 2.5330 & 2.2779 & 2.0445 & 1.8567 \\
\hline 10 & 1.5707 & 1.6907 & 1.8739 & 2.1456 & 2.4672 & 2.5312 & 2.2766 & 2.0440 & 1.8573 \\
\hline 11 & 1.5700 & 1.6897 & 1.8730 & 2.1434 & 2.4634 & 2.5297 & 2.2754 & 2.0436 & 1.8577 \\
\hline 12 & 1.5696 & 1.6889 & 1.8722 & 2.1416 & 2.4606 & 2.5285 & 2.2745 & 2.0433 & 1.8580 \\
\hline 13 & 1.5692 & 1.6883 & 1.8716 & 2.1402 & 2.4583 & 2.5275 & 2.2738 & 2.0430 & 1.8581 \\
\hline 14 & 1.5689 & 1.6879 & 1.8711 & 2.1390 & 2.4564 & 2.5267 & 2.2731 & 2.0428 & 1.8582 \\
\hline 15 & 1.5686 & 1.6876 & 1.8707 & 2.1382 & 2.4550 & 2.5260 & 2.2727 & 2.0427 & 1.8584 \\
\hline 16 & 1.5684 & 1.6873 & 1.8704 & 2.1374 & 2.4538 & 2.5254 & 2.2722 & 2.0425 & 1.8584 \\
\hline 17 & 1.5683 & 1.6870 & 1.8702 & 2.1368 & 2.4528 & 2.5248 & 2.2718 & 2.0424 & 1.8585 \\
\hline 18 & 1.5681 & 1.6867 & 1.8700 & 2.1364 & 2.4518 & 2.5244 & 2.2715 & 2.0423 & 1.8585 \\
\hline 19 & 1.5681 & 1.6866 & 1.8698 & 2.1359 & 2.4511 & 2.5240 & 2.2712 & 2.0422 & 1.8585 \\
\hline 20 & 1.5680 & 1.6864 & 1.8696 & 2.1354 & 2.4505 & 2.5237 & 2.2710 & 2.0422 & 1.8585 \\
\hline 50 & 1.5675 & 1.6852 & 1.8690 & 2.1325 & 2.4460 & 2.5208 & 2.2690 & 2.0416 & 1.8589 \\
\hline 200 & 1.5675 & 1.6851 & 1.8691 & 2.1320 & 2.4454 & 2.5204 & 2.2686 & 2.0416 & 1.8590 \\
\hline
\end{tabular}

Table 10

Frequency parameter $\lambda($ Case $2, c=2, d=14$, SA-1)

\begin{tabular}{|c|c|c|c|c|c|c|c|c|c|}
\hline$k$ & $\Delta=0.1$ & $\Delta=0.2$ & $\Delta=0.3$ & $\Delta=0.4$ & $\Delta=0.5$ & $\Delta=0.6$ & $\Delta=0.7$ & $\Delta=0.8$ & $\Delta=0.9$ \\
\hline 1 & 1.3010 & 1.6837 & 2.0515 & 2.3997 & 2.2367 & 1.8714 & 1.3330 & - & - \\
\hline 2 & 1.1762 & 1.5010 & 1.8307 & 2.1476 & 2.2210 & 1.9609 & 1.6008 & 1.2060 & - \\
\hline 3 & 1.1500 & 1.4637 & 1.7752 & 2.0845 & 2.2333 & 1.9612 & 1.6257 & 1.2596 & - \\
\hline 4 & 1.1404 & 1.4502 & 1.7524 & 2.0596 & 2.2311 & 1.9618 & 1.6380 & 1.2804 & 0.9255 \\
\hline 5 & 1.1358 & 1.4438 & 1.7408 & 2.0478 & 2.2270 & 1.9639 & 1.6451 & 1.2911 & 0.9503 \\
\hline 6 & 1.1336 & 1.4403 & 1.7344 & 2.0416 & 2.2234 & 1.9665 & 1.6494 & 1.2963 & 0.9636 \\
\hline 7 & 1.1320 & 1.4384 & 1.7302 & 2.0379 & 2.2207 & 1.9688 & 1.6520 & 1.2992 & 0.9711 \\
\hline 8 & 1.1311 & 1.4372 & 1.7274 & 2.0353 & 2.2187 & 1.9708 & 1.6538 & 1.3008 & 0.9752 \\
\hline 9 & 1.1307 & 1.4363 & 1.7254 & 2.0336 & 2.2171 & 1.9726 & 1.6550 & 1.3015 & 0.9778 \\
\hline 10 & 1.1303 & 1.4358 & 1.7239 & 2.0324 & 2.2160 & 1.9741 & 1.6557 & 1.3021 & 0.9793 \\
\hline 11 & 1.1300 & 1.4354 & 1.7228 & 2.0314 & 2.2149 & 1.9755 & 1.6564 & 1.3025 & 0.9803 \\
\hline 12 & 1.1298 & 1.4353 & 1.7219 & 2.0306 & 2.2142 & 1.9766 & 1.6568 & 1.3027 & 0.9808 \\
\hline 13 & 1.1298 & 1.4351 & 1.7212 & 2.0301 & 2.2135 & 1.9776 & 1.6573 & 1.3029 & 0.9813 \\
\hline 14 & 1.1296 & 1.4349 & 1.7206 & 2.0298 & 2.2129 & 1.9785 & 1.6576 & 1.3029 & 0.9818 \\
\hline 15 & 1.1296 & 1.4347 & 1.7202 & 2.0294 & 2.2125 & 1.9793 & 1.6577 & 1.3031 & 0.9818 \\
\hline 16 & 1.1296 & 1.4347 & 1.7197 & 2.0293 & 2.2120 & 1.9799 & 1.6580 & 1.3031 & 0.9821 \\
\hline 17 & 1.1296 & 1.4346 & 1.7194 & 2.0290 & 2.2117 & 1.9805 & 1.6582 & 1.3031 & 0.9823 \\
\hline 18 & 1.1296 & 1.4346 & 1.7192 & 2.0289 & 2.2113 & 1.9810 & 1.6583 & 1.3031 & 0.9823 \\
\hline 19 & 1.1296 & 1.4346 & 1.7189 & 2.0288 & 2.2111 & 1.9815 & 1.6585 & 1.3031 & 0.9823 \\
\hline 20 & 1.1296 & 1.4344 & 1.7187 & 2.0288 & 2.2109 & 1.9819 & 1.6586 & 1.3031 & 0.9823 \\
\hline 50 & 1.1296 & 1.4342 & 1.7167 & 2.0288 & 2.2087 & 1.9868 & 1.6595 & 1.3033 & 0.9826 \\
\hline 200 & 1.1298 & 1.4342 & 1.7164 & 2.0290 & 2.2083 & 1.9881 & 1.6597 & 1.3033 & 0.9826 \\
\hline
\end{tabular}


Table 11

Frequency parameter $\lambda($ Case $2, c=2, d=14, \mathrm{AS}-1)$

\begin{tabular}{|c|c|c|c|c|c|c|c|c|c|}
\hline$k$ & $\Delta=0.1$ & $\Delta=0.2$ & $\Delta=0.3$ & $\Delta=0.4$ & $\Delta=0.5$ & $\Delta=0.6$ & $\Delta=0.7$ & $\Delta=0.8$ & $\Delta=0.9$ \\
\hline 1 & 0.8334 & 0.9423 & 1.1156 & 1.3784 & 1.4891 & 1.2106 & 0.9905 & - & - \\
\hline 2 & 0.7106 & 0.7944 & 0.9287 & 1.1338 & 1.3869 & 1.2544 & 1.0529 & 0.8741 & - \\
\hline 3 & 0.6786 & 0.7550 & 0.8769 & 1.0677 & 1.3265 & 1.2568 & 1.0696 & 0.8983 & - \\
\hline 4 & 0.6656 & 0.7389 & 0.8556 & 1.0409 & 1.2969 & 1.2564 & 1.0759 & 0.9097 & 0.7540 \\
\hline 5 & 0.6592 & 0.7311 & 0.8447 & 1.0271 & 1.2808 & 1.2558 & 1.0789 & 0.9152 & 0.7642 \\
\hline 6 & 0.6554 & 0.7263 & 0.8385 & 1.0196 & 1.2708 & 1.2556 & 1.0805 & 0.9179 & 0.7704 \\
\hline 7 & 0.6531 & 0.7235 & 0.8343 & 1.0146 & 1.2645 & 1.2556 & 1.0814 & 0.9195 & 0.7743 \\
\hline 8 & 0.6515 & 0.7218 & 0.8316 & 1.0114 & 1.2600 & 1.2554 & 1.0819 & 0.9203 & 0.7769 \\
\hline 9 & 0.6504 & 0.7204 & 0.8298 & 1.0092 & 1.2570 & 1.2554 & 1.0824 & 0.9209 & 0.7785 \\
\hline 10 & 0.6496 & 0.7194 & 0.8283 & 1.0075 & 1.2546 & 1.2554 & 1.0826 & 0.9211 & 0.7791 \\
\hline 11 & 0.6492 & 0.7187 & 0.8273 & 1.0062 & 1.2528 & 1.2556 & 1.0826 & 0.9214 & 0.7797 \\
\hline 12 & 0.6488 & 0.7180 & 0.8264 & 1.0055 & 1.2514 & 1.2556 & 1.0828 & 0.9214 & 0.7804 \\
\hline 13 & 0.6485 & 0.7176 & 0.8258 & 1.0047 & 1.2504 & 1.2556 & 1.0828 & 0.9217 & 0.7807 \\
\hline 14 & 0.6481 & 0.7173 & 0.8255 & 1.0042 & 1.2496 & 1.2556 & 1.0831 & 0.9217 & 0.7807 \\
\hline 15 & 0.6481 & 0.7173 & 0.8249 & 1.0037 & 1.2488 & 1.2558 & 1.0831 & 0.9217 & 0.7810 \\
\hline 16 & 0.6477 & 0.7169 & 0.8246 & 1.0032 & 1.2482 & 1.2558 & 1.0831 & 0.9217 & 0.7810 \\
\hline 17 & 0.6477 & 0.7166 & 0.8243 & 1.0030 & 1.2478 & 1.2558 & 1.0831 & 0.9217 & 0.7813 \\
\hline 18 & 0.6477 & 0.7166 & 0.8240 & 1.0027 & 1.2474 & 1.2558 & 1.0833 & 0.9217 & 0.7813 \\
\hline 19 & 0.6473 & 0.7166 & 0.8240 & 1.0025 & 1.2470 & 1.2560 & 1.0833 & 0.9220 & 0.7813 \\
\hline 20 & 0.6473 & 0.7162 & 0.8237 & 1.0022 & 1.2466 & 1.2560 & 1.0833 & 0.9220 & 0.7813 \\
\hline 50 & 0.6469 & 0.7155 & 0.8225 & 1.0010 & 1.2444 & 1.2566 & 1.0833 & 0.9220 & 0.7817 \\
\hline 200 & 0.6469 & 0.7155 & 0.8222 & 1.0007 & 1.2440 & 1.2568 & 1.0833 & 0.9220 & 0.7817 \\
\hline
\end{tabular}

Table 12

Frequency parameter $\lambda($ Case $2, c=2, d=15$, AA-1)

\begin{tabular}{|c|c|c|c|c|c|c|c|c|c|}
\hline$k$ & $\Delta=0.1$ & $\Delta=0.2$ & $\Delta=0.3$ & $\Delta=0.4$ & $\Delta=0.5$ & $\Delta=0.6$ & $\Delta=0.7$ & $\Delta=0.8$ & $\Delta=0.9$ \\
\hline 1 & 0.4207 & 0.7043 & 1.0102 & 1.3620 & 1.8727 & 2.3160 & 1.8108 & - & - \\
\hline 2 & 0.3413 & 0.5736 & 0.8056 & 1.1057 & 1.4844 & 1.9927 & 1.9865 & 1.6213 & - \\
\hline 3 & 0.3309 & 0.5477 & 0.7649 & 1.0452 & 1.3855 & 1.8585 & 2.0116 & 1.6630 & - \\
\hline 4 & 0.3279 & 0.5371 & 0.7497 & 1.0196 & 1.3426 & 1.8040 & 2.0200 & 1.6794 & 1.3388 \\
\hline 5 & 0.3263 & 0.5315 & 0.7423 & 1.0062 & 1.3206 & 1.7758 & 2.0224 & 1.6857 & 1.3594 \\
\hline 6 & 0.3256 & 0.5287 & 0.7382 & 0.9985 & 1.3081 & 1.7594 & 2.0226 & 1.6883 & 1.3691 \\
\hline 7 & 0.3256 & 0.5263 & 0.7355 & 0.9932 & 1.3002 & 1.7489 & 2.0223 & 1.6897 & 1.3740 \\
\hline 8 & 0.3256 & 0.5254 & 0.7342 & 0.9899 & 1.2950 & 1.7420 & 2.0216 & 1.6903 & 1.3766 \\
\hline 9 & 0.3256 & 0.5244 & 0.7328 & 0.9874 & 1.2913 & 1.7371 & 2.0210 & 1.6907 & 1.3780 \\
\hline 10 & 0.3256 & 0.5239 & 0.7321 & 0.9856 & 1.2888 & 1.7335 & 2.0205 & 1.6912 & 1.3789 \\
\hline 11 & 0.3256 & 0.5235 & 0.7318 & 0.9844 & 1.2869 & 1.7309 & 2.0200 & 1.6913 & 1.3795 \\
\hline 12 & 0.3256 & 0.5230 & 0.7314 & 0.9834 & 1.2853 & 1.7290 & 2.0197 & 1.6914 & 1.3800 \\
\hline 13 & 0.3256 & 0.5225 & 0.7311 & 0.9823 & 1.2841 & 1.7274 & 2.0193 & 1.6916 & 1.3804 \\
\hline 14 & 0.3256 & 0.5225 & 0.7308 & 0.9818 & 1.2834 & 1.7261 & 2.0190 & 1.6917 & 1.3808 \\
\hline 15 & 0.3256 & 0.5220 & 0.7308 & 0.9813 & 1.2826 & 1.7251 & 2.0188 & 1.6917 & 1.3809 \\
\hline 16 & 0.3256 & 0.5220 & 0.7308 & 0.9808 & 1.2820 & 1.7244 & 2.0187 & 1.6919 & 1.3811 \\
\hline 17 & 0.3256 & 0.5220 & 0.7304 & 0.9806 & 1.2814 & 1.7237 & 2.0184 & 1.6919 & 1.3813 \\
\hline 18 & 0.3256 & 0.5220 & 0.7304 & 0.9801 & 1.2810 & 1.7231 & 2.0183 & 1.6920 & 1.3815 \\
\hline 19 & 0.3256 & 0.5215 & 0.7304 & 0.9798 & 1.2806 & 1.7226 & 2.0182 & 1.6920 & 1.3815 \\
\hline 20 & 0.3256 & 0.5215 & 0.7304 & 0.9795 & 1.2804 & 1.7222 & 2.0180 & 1.6920 & 1.3817 \\
\hline 50 & 0.3263 & 0.5211 & 0.7304 & 0.9778 & 1.2785 & 1.7194 & 2.0171 & 1.6925 & 1.3826 \\
\hline 200 & 0.3263 & 0.5211 & 0.7304 & 0.9775 & 1.2781 & 1.7192 & 2.0169 & 1.6926 & 1.3828 \\
\hline
\end{tabular}


Table 13

Frequency parameter $\lambda($ Case $2, c=1)$

\begin{tabular}{|c|c|c|c|c|c|c|c|c|c|c|c|c|}
\hline \multirow{3}{*}{$\begin{array}{c}c=1 \\
k\end{array}$} & \multicolumn{6}{|c|}{$\Delta=0.5$} & \multicolumn{6}{|c|}{$\Delta=0.9$} \\
\hline & \multicolumn{2}{|c|}{$d=15$} & \multicolumn{2}{|c|}{$d=14$} & \multicolumn{2}{|c|}{$d=15$} & \multicolumn{2}{|c|}{$d=15$} & \multicolumn{2}{|c|}{$d=14$} & \multicolumn{2}{|c|}{$d=15$} \\
\hline & SS-2 & SS-3 & SA-2 & SA-3 & AA-2 & AA-3 & SS-2 & SS-3 & SA-2 & SA-3 & AA-2 & AA-3 \\
\hline 1 & 2.3173 & 3.0868 & 1.8829 & 2.9579 & 2.2051 & 2.9977 & - & - & - & - & - & - \\
\hline 2 & 2.1617 & 2.2521 & 1.5511 & 2.6300 & 2.0822 & 2.7048 & - & - & - & - & - & - \\
\hline 3 & 2.0214 & 2.2396 & 1.5427 & 2.5089 & 2.0276 & 2.6525 & - & - & - & - & - & - \\
\hline 4 & 1.9834 & 2.2319 & 1.5505 & 2.4552 & 2.0019 & 2.6382 & 2.0491 & 2.3495 & 1.7710 & 2.1677 & 1.6216 & 2.5963 \\
\hline 5 & 1.9690 & 2.2270 & 1.5535 & 2.4267 & 1.9881 & 2.6330 & 2.0580 & 2.3865 & 1.7909 & 2.1798 & 1.6397 & 2.5941 \\
\hline 6 & 1.9621 & 2.2238 & 1.5550 & 2.4096 & 1.9800 & 2.6309 & 2.0645 & 2.4025 & 1.8000 & 2.1875 & 1.6523 & 2.5930 \\
\hline 7 & 1.9584 & 2.2217 & 1.5560 & 2.3982 & 1.9751 & 2.6298 & 2.0685 & 2.4086 & 1.8040 & 2.1919 & 1.6603 & 2.5922 \\
\hline 8 & 1.9563 & 2.2202 & 1.5564 & 2.3905 & 1.9718 & 2.6294 & 2.0709 & 2.4107 & 1.8060 & 2.1947 & 1.6651 & 2.5916 \\
\hline 9 & 1.9550 & 2.2191 & 1.5569 & 2.3848 & 1.9695 & 2.6291 & 2.0724 & 2.4114 & 1.8068 & 2.1962 & 1.6681 & 2.5911 \\
\hline 10 & 1.9542 & 2.2183 & 1.5571 & 2.3807 & 1.9679 & 2.6289 & 2.0734 & 2.4117 & 1.8072 & 2.1974 & 1.6700 & 2.5908 \\
\hline 11 & 1.9537 & 2.2177 & 1.5572 & 2.3775 & 1.9667 & 2.6288 & 2.0740 & 2.4119 & 1.8073 & 2.1982 & 1.6712 & 2.5906 \\
\hline 12 & 1.9533 & 2.2172 & 1.5574 & 2.3751 & 1.9658 & 2.6287 & 2.0745 & 2.4122 & 1.8075 & 2.1986 & 1.6721 & 2.5904 \\
\hline 13 & 1.9532 & 2.2169 & 1.5574 & 2.3732 & 1.9651 & 2.6286 & 2.0747 & 2.4124 & 1.8076 & 2.1990 & 1.6727 & 2.5902 \\
\hline 14 & 1.9531 & 2.2165 & 1.5574 & 2.3716 & 1.9644 & 2.6286 & 2.0750 & 2.4125 & 1.8078 & 2.1993 & 1.6732 & 2.5900 \\
\hline 15 & 1.9529 & 2.2163 & 1.5574 & 2.3703 & 1.9641 & 2.6286 & 2.0751 & 2.4127 & 1.8078 & 2.1995 & 1.6735 & 2.5899 \\
\hline 16 & 1.9529 & 2.2161 & 1.5574 & 2.3693 & 1.9637 & 2.6286 & 2.0752 & 2.4128 & 1.8078 & 2.1997 & 1.6738 & 2.5898 \\
\hline 17 & 1.9528 & 2.2160 & 1.5574 & 2.3683 & 1.9634 & 2.6285 & 2.0753 & 2.4129 & 1.8079 & 2.1998 & 1.6739 & 2.5897 \\
\hline 18 & 1.9528 & 2.2159 & 1.5574 & 2.3676 & 1.9632 & 2.6285 & 2.0755 & 2.4130 & 1.8079 & 2.1998 & 1.6741 & 2.5896 \\
\hline 19 & 1.9528 & 2.2157 & 1.5574 & 2.3670 & 1.9629 & 2.6285 & 2.0755 & 2.4131 & 1.8079 & 2.1999 & 1.6742 & 2.5895 \\
\hline 20 & 1.9529 & 2.2156 & 1.5574 & 2.3664 & 1.9627 & 2.6285 & 2.0755 & 2.4131 & 1.8079 & 2.1999 & 1.6744 & 2.5895 \\
\hline 50 & 1.9536 & 2.2152 & 1.5574 & 2.3622 & 1.9615 & 2.6285 & 2.0761 & 2.4137 & 1.8079 & 2.2000 & 1.6757 & 2.5889 \\
\hline 200 & 1.9538 & 2.2151 & 1.5574 & 2.3616 & 1.9614 & 2.6285 & 2.0763 & 2.4139 & 1.8079 & 2.2000 & 1.6762 & 2.5888 \\
\hline $\begin{array}{c}\text { Ref. } \\
{[1]}\end{array}$ & 1.9190 & 2.1900 & 2.3280 & & & & 2.0710 & 2.4050 & 2.1880 & & & \\
\hline
\end{tabular}

Table 14

Fundamental mode

\begin{tabular}{ccccc}
\hline Case no & $c$ & Interval of $k$ & Support location & Fundamental mode \\
\hline 1 & 1 & $k=1$ & - & SS-1 AA-1 \\
1 & 1 & $2 \leqslant k \leqslant 200$ & - & AA-1 \\
1 & 2 & $1 \leqslant k \leqslant 200$ & - & AS-1 \\
2 & 1 & $1 \leqslant k \leqslant 200$ & $0.1 \leqslant \Delta \leqslant 0.4$ & AA-1 \\
2 & 1 & $k=1$ & $\Delta=0.5$ & SA-1 \\
2 & 1 & $2 \leqslant k \leqslant 200$ & $\Delta=0.5$ & AA-1 \\
2 & 1 & $1 \leqslant k \leqslant 200$ & $0.6 \leqslant \Delta \leqslant 0.9$ & AS-1 \\
2 & 2 & $1 \leqslant k \leqslant 200$ & $0.1 \leqslant \Delta \leqslant 0.4$ & AA-1 \\
2 & 2 & $1 \leqslant k \leqslant 200$ & $0.5 \leqslant \Delta \leqslant 0.9$ & AS-1
\end{tabular}

Table 15

Optimum support location (Case 2)

\begin{tabular}{ccc}
\hline$c$ & Interval of $k$ & Support location \\
\hline 1 & $1 \leqslant k \leqslant 2$ & $\Delta=0.5$ \\
1 & $3 \leqslant k \leqslant 200$ & $\Delta=0.6$ \\
2 & $1 \leqslant k \leqslant 9$ & $\Delta=0.5$ \\
2 & $10 \leqslant k \leqslant 200$ & $\Delta=0.6$ \\
\hline
\end{tabular}

distinct aspect ratios were considered in the numerical computations which were carried out for two cases: (1) a completely free plate, (2) a plate with four intermediate point-supports symmetrically distributed about the central axes of the plate.

The results obtained in the current study were compared with those of rectangular plates by setting $k=200$. For a completely free plate, almost identical results were obtained (Tables 3-5). For the point-supported case good 

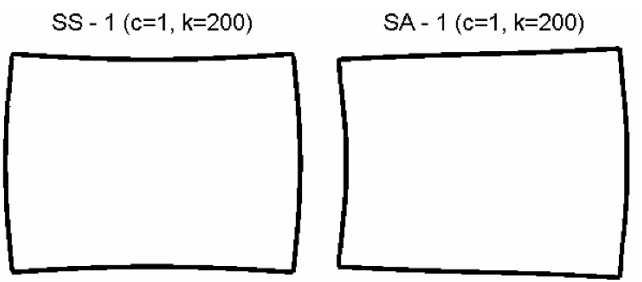

AS - $1(c=1, k=200)$

$A A-1(c=1, k=200)$
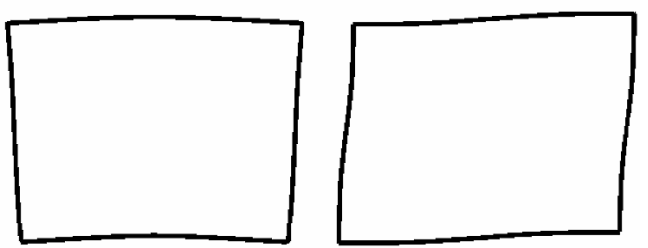

Fig. 2. Mode shapes ( $1^{\text {st }}$ mode, Case $\left.1, c=1\right)$.

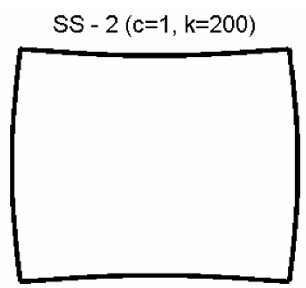

$S A-2(c=1, k=200)$

AS - $2(c=1, k=200)$
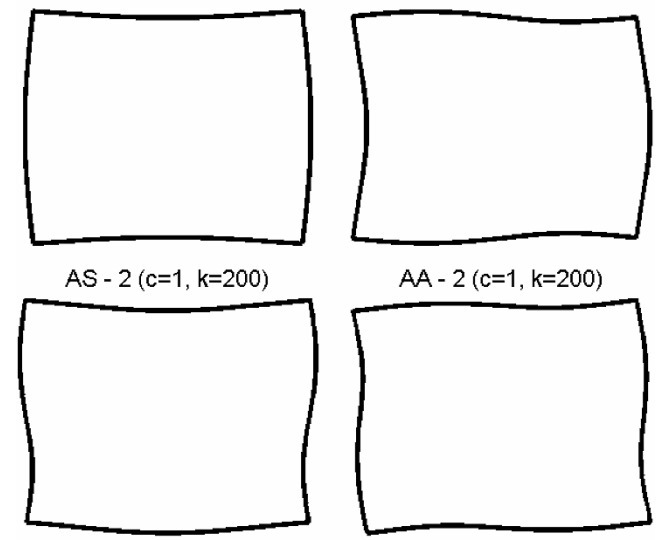

$\mathrm{AA}-2(\mathrm{c}=1, \mathrm{k}=200)$

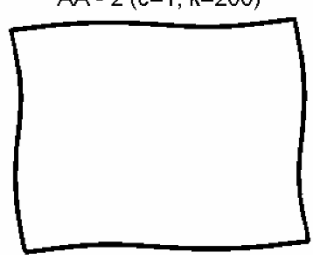

Fig. 3. Mode shapes ( $2^{\text {nd }}$ mode, Case $\left.1, c=1\right)$.

SS $-3(c=1, k=200)$

$S A-3(c=1, k=200)$

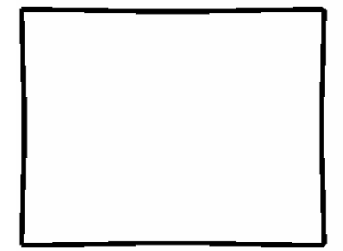

AS $-3(c=1, k=200)$

$\mathrm{AA}-3(\mathrm{c}=1, \mathrm{k}=200)$
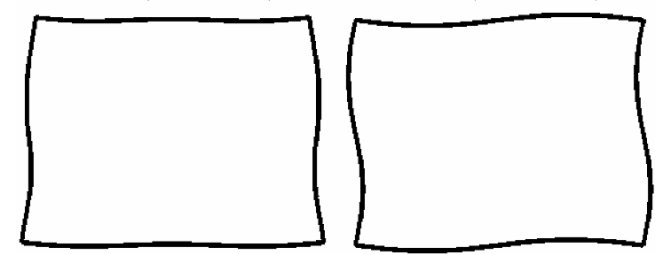

Fig. 4. Mode shapes $\left(3^{r d}\right.$ mode, Case $\left.1, c=1\right)$. 


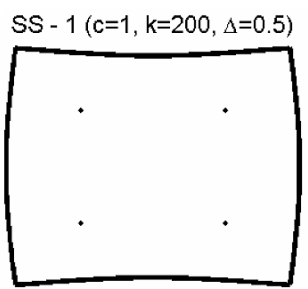

$\mathrm{SA}-1(\mathrm{c}=1, \mathrm{k}=200, \Delta=0.5)$

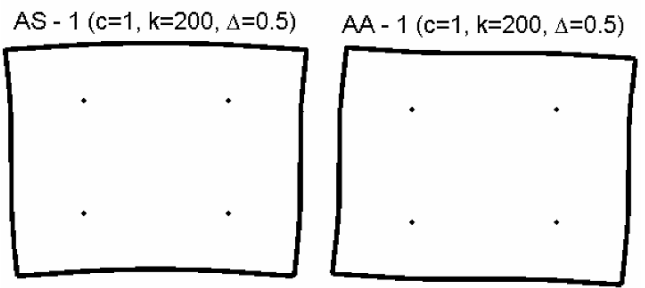

Fig. 5. Mode shapes ( $1^{\text {st }}$ mode, Case $2, c=1, \Delta=0.5$ ).

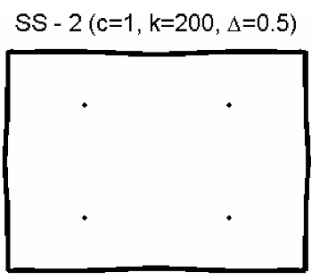

$\mathrm{SA}-2(\mathrm{c}=1, \mathrm{k}=200, \Delta=0.5)$

AS - $2(\mathrm{c}=1, \mathrm{k}=200, \Delta=0.5)$
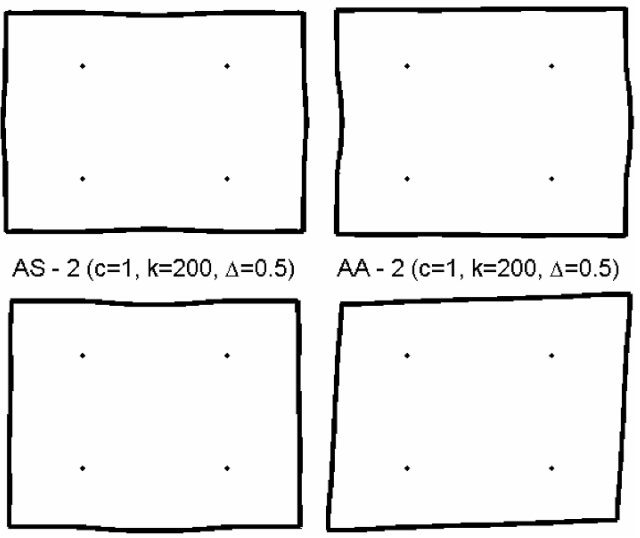

AA - $2(\mathrm{c}=1, \mathrm{k}=200, \Delta=0.5)$

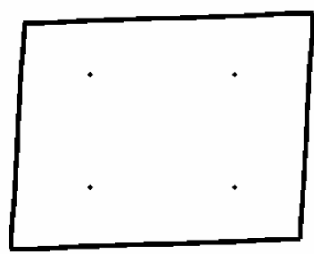

Fig. 6. Mode shapes ( $2^{n d}$ mode, Case $2, c=1, \Delta=0.5$ ).

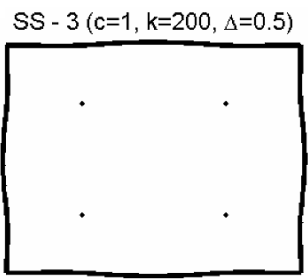

SA $-3(c=1, k=200, \Delta=0.5)$

AS - $3(\mathrm{c}=1, \mathrm{k}=200, \Delta=0.5)$

$\mathrm{AA}-3(\mathrm{c}=1, \mathrm{k}=200, \Delta=0.5)$
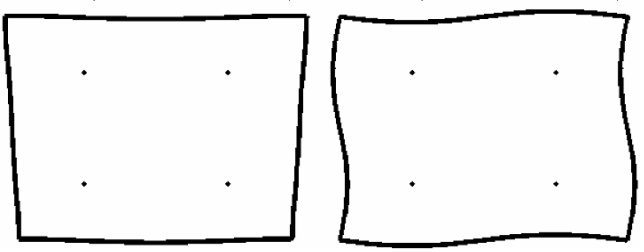

Fig. 7. Mode shapes ( $3^{r d}$ mode, Case $\left.2, c=1, \Delta=0.5\right)$. 
agreement was observed for SS modes (Tables 6, 13), but lower eigenvalues were computed for SA, and AA modes when $\Delta=0.5$ and $\Delta=0.9$. Interestingly, the frequencies corresponding to the second and the third SA and AA modes obtained in this paper seem to be in harmony with those corresponding to the first and the second modes reported in Ref. [1] (Tables 7-8, 13). The convergence study was presented (Table 2), and the mode shapes were depicted as an example (Figs 2-7).

The mode corresponding to the fundamental frequency for each case was determined. It was shown that AA-1 and AS-1 modes generally yield the fundamental frequency (Table 14). The optimum support location for a pointsupported super-elliptical plate was presented. It can be concluded that for $k>9$ the optimum support location is $\Delta=0.6$ (Table 15).

\section{References}

[1] Y. Kobayashi, G. Yamada and S. Honma, In-plane vibration of point-supported rectangular plates, Journal of Sound and Vibration 126(3) (1988), 545-549.

[2] N.S. Bardell, R.S. Langley and J.M. Dunsdon, On the free in-plane vibration of isotropic rectangular plates, Journal of Sound and Vibration 191(3) (1996), 459-467.

[3] K. Hyde, J.Y. Chang, C. Bacca and J.A. Wickert, Parameter studies for plane stress in-plane vibration of rectangular plates, Journal of Sound and Vibration 247(3) (2001), 471-487.

[4] J. Du, W.L. Li, G. Jin, T. Yang and Z. Liu, An analytical method for the in-plane vibration analysis of rectangular plates with elastically restrained edges, Journal of Sound and Vibration 306 (2007), 908-927.

[5] D.J. Gorman, Free in-plane vibration analysis of rectangular plates by the method of superposition, Journal of Sound and Vibration 272 (2004), 831-851.

[6] D.J. Gorman, Free in-plane vibration analysis of rectangular plates with elastic support normal to the boundaries, Journal of Sound and Vibration 285 (2005), 941-966.

[7] N.H. Farag and J. Pan, Modal characteristics of in-plane vibration of rectangular plates, Journal of the Acoustical Society of America 105(6) (1999), 3295-3310.

[8] N.H. Farag and J. Pan, Free and forced in-plane vibration of rectangular plates, Journal of the Acoustical Society of America 103(1) (1998), $408-413$.

[9] B.A. Ovunc, In-plane vibration of plates under external disturbances applied at singular points, Thin-Walled Structures 18(1994), 83-95.

[10] T. Irie, G. Yamada and Y. Muramoto, Natural frequencies of in-plane vibration of annular plates, Journal of Sound and Vibration 97(1) (1984), 171-175.

[11] A.N. Bercin, An assessment of the effects of in-plane vibrations on the energy flow between coupled plates, Journal of Sound and Vibration 191(5) (1996), 661-680.

[12] N.H. Farag and J. Pan, Modal characteristics of in-plane vibration of circular plates clamped at the outer edge, Journal of the Acoustical Society of America 113(4) (2003), 1935-1946.

[13] A.N. Bercin and R.S. Langley, Application of the dynamic stiffness technique to the in-plane vibrations of plate structures, Computers and Structures 59(5) (1996), 869-875.

[14] B. Ovunc, In-plane vibration of plates by continuous mass matrix method, Computers and Structures 8 (1978), $723-731$.

[15] A.V. Singh and T. Muhammad, Free in-plane vibration of isotropic non-rectangular plates, Journal of Sound and Vibration 273 (2004), 219-231.

[16] C.I. Park, Frequency equation for the in-plane vibration of a clamped circular plate, Journal of Sound and Vibration 313(1-2) (2008), $325-333$.

[17] I.V. Andrianov, J. Awrejcewicz and V. Chernetskyy, Analysis of natural in-plane vibration of rectangular plates using homotopy perturbation approach, Mathematical Problems in Engineering (2006), DOI 10.1155/MPE/2006/20598 1-8.

[18] D.C. Chang, G. Wang and N.M. Wereley, A generalized Kantorovich method and its application to free in-plane plate vibration problem, Applicable Analysis 80(3) (2001), 493-523.

[19] A. Houmat, In-plane vibration of plates with curvilinear plan-forms by a trigonometrically enriched curved triangular p-element, ThinWalled Structures 46(2) (2008), 103-111.

[20] A.R.H. Henni, C. Bacon and Liu, In-plane vibration of thin elliptic plates submitted to uniform pulsed microwave irradiations, Journal of Sound and Vibration 299 (2007), 298-313.

[21] G. Wang and N.M. Wereley, Free in-plane vibration of rectangular plates, AIAA Journal 40(5) (2002), 953-959.

[22] R.H. Gutierrez and P.A.A. Laura, In-plane vibrations of thin, elastic rectangular plates elastically restrained against translation along the edges, Journal of Sound and Vibration 132(3) (1989), 512-515.

[23] D. Larsson, In-plane modal testing of a free isotropic rectangular plate, Experimental Mechanics 37(3) (1997), 339-343.

[24] D.J. Gorman, Accurate analytical type solutions for the free in-plane vibration of clamped and simply supported rectangular plates, Journal of Sound and Vibration 276 (2004), 311-333.

[25] D.J. Gorman, Exact solutions for the free in-plane vibration of rectangular plates with two opposite edges simply supported, Journal of Sound and Vibration 294 (2006), 131-161.

[26] D.C. Gazis and R.D. Mindlin, Extensional vibrations and waves in a circular disk and a semi-infinite plate, Journal of Applied Mechanics (1960), 541-547. 
[27] S.S.H. Chen and T.M. Liu, Extensional vibration of thin plates of various shapes, Journal of the Acoustical Society of America 58(4) (1975), 828-831.

[28] R. Kirisik and S. Yuksel, Free vibration analysis of a rectangular plate with Kelvin type boundary conditions, Shock and Vibration 14 (2007), 90-95.

[29] W.J. Si, K.Y. Lam and S.W. Gong, Vibration analysis of rectangular plates with one or more guided edges via bicubic B-spline method, Shock and Vibration 12(5) (2005), 363-376.

[30] M. Altekin, Free vibration of orthotropic super-elliptical plates on intermediate supports, Nuclear Engineering and Design 239(6) (2009), 981-999.

[31] L. Wu and J. Liu, Free vibration analysis of arbitrary shaped thick plates by differential cubature method, International Journal of Mechanical Sciences 47 (2005), 63-81.

[32] C.C. Chen, C.W. Lim, S. Kitipornchai and K.M. Liew, Vibration of symmetrically laminated thick super elliptical plates, Journal of Sound and Vibration 220(4) (1999), 659-682.

[33] K.M. Liew and Z.C. Feng, Three-dimensional free vibration analysis of perforated superelliptical plates via the p-Ritz method, International Journal of Mechanical Sciences 43 (2001), 2613-2630.

[34] C.W. Lim and K.M. Liew, Vibrations of perforated plates with rounded corners, Journal of Engineering Mechanics 121(2) (1995), $203-213$.

[35] C.M. Wang, L. Wang and K.M. Liew, Vibration and buckling of super elliptical plates, Journal of Sound and Vibration 171(3) (1994), 301-314.

[36] D. Zhou, S.H. Lo, Y.K. Cheung and F.T.K. Au, 3-D vibration analysis of generalized super elliptical plates using Chebyshev-Ritz method, International Journal of Solids and Structures 41 (2004), 4697-4712.

[37] C.W. Lim, S. Kitipornchai and K.M. Liew, A free-vibration analysis of doubly connected super-elliptical laminated composite plates, Composites Science and Technology 58 (1998), 435-445.

[38] K.M. Liew, S. Kitipornchai and C.W. Lim, Free vibration analysis of thick superelliptical plates, Journal of Engineering Mechanics 124(2) (1998), 137-145.

[39] D.J. Gorman, Free-vibration analysis of point-supported orthotropic plates, Journal of Engineering Mechanics 120(1) (1994), 58-74.

[40] C.A. Brebbia, The Boundary Element Method for Engineers, Pentech Press, London, 1984. 

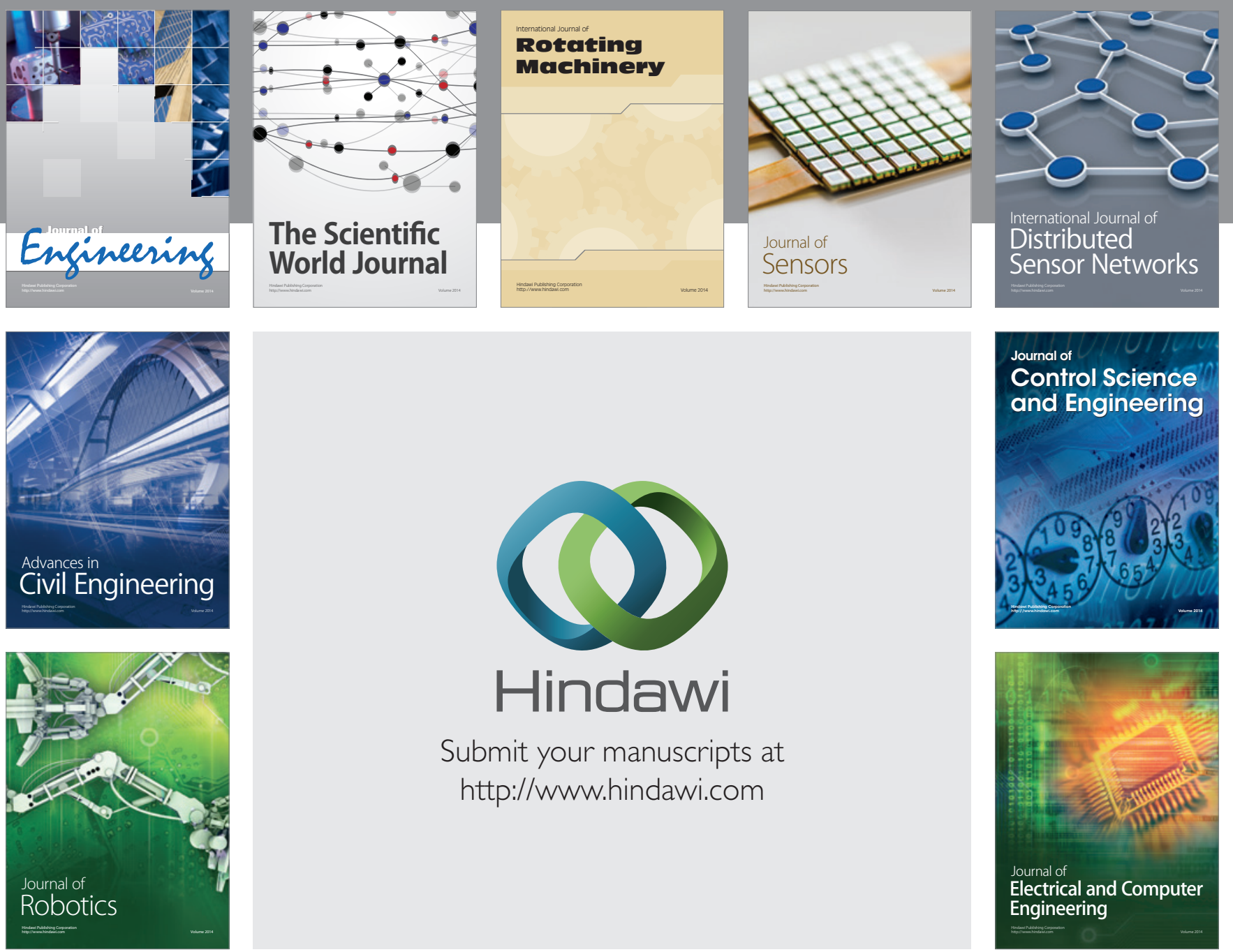

Submit your manuscripts at

http://www.hindawi.com
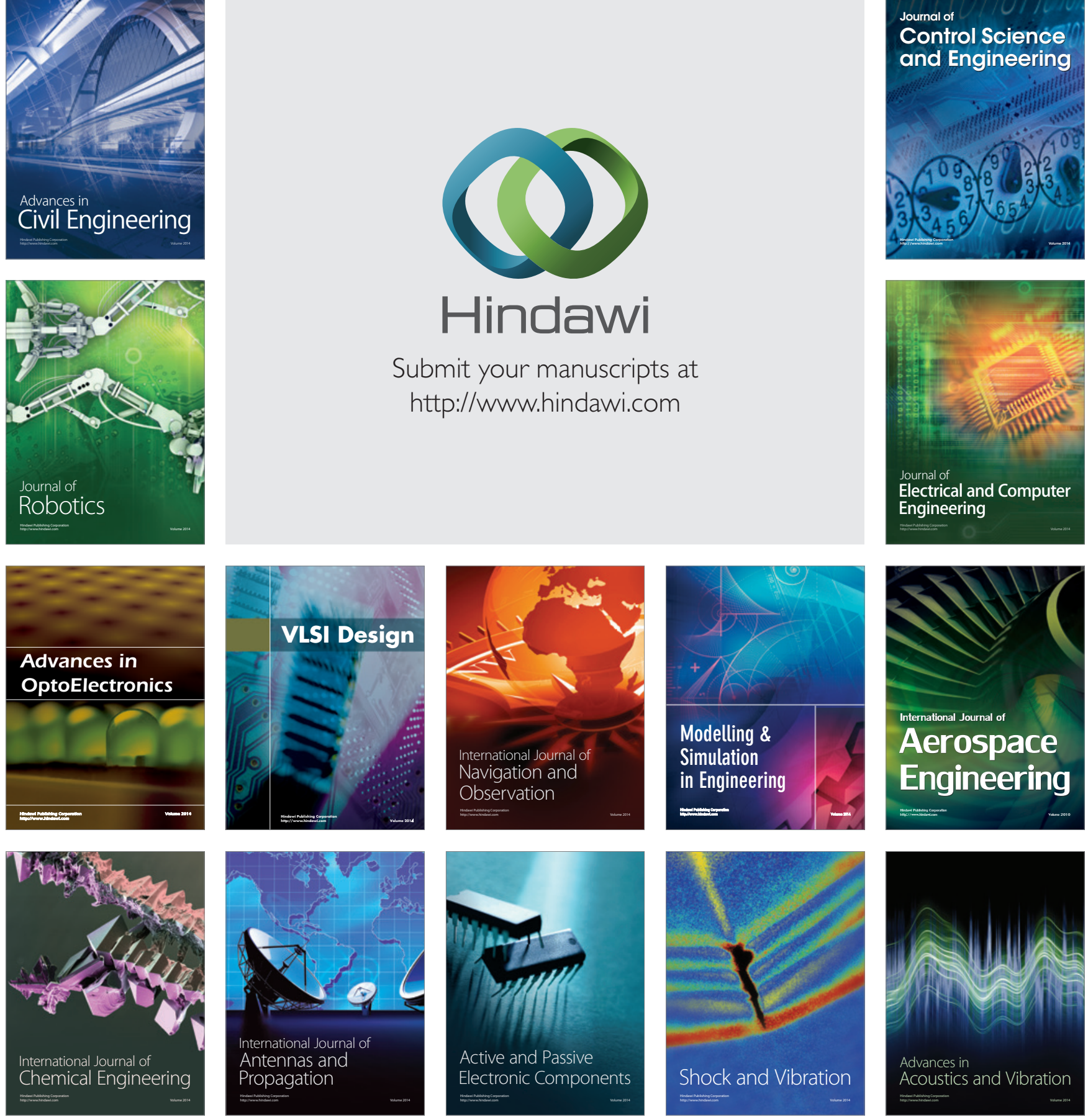